\title{
Stylistique(s) contrastive(s) du discours scientifique
}

Michel Petit

\section{(2) OpenEdition}

\section{Journals}

Édition électronique

URL : http://journals.openedition.org/asp/3015

DOI : 10.4000/asp.3015

ISSN : 2108-6354

\section{Éditeur}

Groupe d'étude et de recherche en anglais de spécialité

\section{Édition imprimée}

Date de publication : 1 décembre 1997

Pagination : 139-156

ISSN : 1246-8185

\section{Référence électronique}

Michel Petit, «Stylistique(s) contrastive(s) du discours scientifique », ASp [En ligne], 15-18| 1997, mis en ligne le 25 mai 2012, consulté le 01 mai 2019. URL : http://journals.openedition.org/asp/3015 ; DOI : 10.4000/asp.3015

Ce document a été généré automatiquement le 1 mai 2019.

Tous droits réservés 


\title{
Stylistique(s) contrastive(s) du discours scientifique
}

\author{
Michel Petit
}

\section{Introduction}

1 À la question « Quelle stylistique pour l'étude du discours scientifique en anglais? », cette réflexion se propose d'apporter des éléments de réponse fondés sur une démarche qui se veut de linguistique. Ce préalable essentiel est loin d'aller de soi, et l'on sait bien que la question des rapports de la stylistique avec la linguistique, considérée "comme une discipline dans le champ des autres disciplines linguistiques » (Molinié 1993 : 2), constitue elle-même un topos de la littérature stylistique ${ }^{1}$. On n'élaborera pas directement ici une réflexion argumentée sur ce préalable, mais on reconnaîtra indirectement dans les orientations proposées quelques-uns des points de ce que pourrait être une telle argumentation.

2 Il faut, après cette ouverture qui tient à l'évidence de la pétition de principe, tenter de définir plus précisément l'ambition de cette étude. L'objet d'étude est le discours scientifique, conçu comme l'ensemble des productions textuelles relevant du domaine des sciences, au sens courant qui correspond dans la tradition institutionnelle aux disciplines présentes dans les facultés des sciences, et ressortissant ipso facto à un «style scientifique » (à définir). Ces productions textuelles sont, comme on le verra, de nature diverse, et c'est en ce premier sens que l'approche se veut contrastive, tentant de déterminer notamment les incidences stylistiques de la catégorisation textuelle à l'intérieur d'une typologie discursive. Mais le terme de "contrastif» fait aussi, classiquement, référence à la comparaison entre langues, ici l'anglais et le français. On évoquera à cet égard le statut qu'il convient de donner aux spécificités proprement stylistiques de toute production textuelle de discours scientifique dans le cadre d'une analyse de linguistique contrastive. 
On est naturellement conscient que l'usage de l'adjectif « stylistique » dans ce qui précède n'éclaire nullement, pour l'instant, la question de ce que peut être la dimension «stylistique » du discours scientifique. Il faut donc, tout d'abord, poser comme principe qu'il existe une dimension stylistique pertinente pour l'étude de toute production textuelle, y compris non littéraire. On songera à la conception selon laquelle

l'objet de la stylistique n'est pas d'abord le style, contrairement à ce qu'on pourrait spontanément penser, même si, en revanche, le style peut difficilement s'appréhender autrement que comme objet d'étude de la stylistique (on en reparlera) : l'objet majeur et éminent de la stylistique, c'est le discours littéraire, la littérature. Plus exactement, c'est le caractère spécifique de littérarité du discours, de la praxis langagière telle qu'elle est concrètement développée, réalisée, à travers un régime bien particulier de fonctionnement du langage, la littérature. (Molinié $1993: 1-2)$

et on suggérera que cette conception, qui définit dans sa formulation la stylistique « littéraire ", peut s'appliquer, mutatis mutandis, à une stylistique qui s'intéresserait au discours scientifique, c'est-à-dire au caractère spécifique de cette praxis langagière concrètement développée à travers le régime de fonctionnement du langage qui est celui du discours scientifique ${ }^{2}$.

\section{Le discours scientifique}

\subsection{Du générique au spécifique}

5 Ce que l'on constate tout d'abord, à l'examen du discours scientifique, même au sens restreint que nous lui attribuons ici, c'est sa diversité. Nous proposons dans cette partie une classification de ces facteurs de diversité, qui concernent notamment :

6 (a) Le domaine de connaissance (mathématiques, chimie, biologie, etc.) et le sousdomaine. La spécificité de chaque domaine se manifeste de façon évidente dans le lexique spécialisé utilisé, mais aussi dans un certain nombre de caractéristiques formelles. On constate ainsi que la nature, la fréquence et l'organisation des notations symboliques et éléments péri-textuels (non strictement textuels) varient de façon significative et essentielle d'un domaine à l'autre et d'un sous-domaine à l'autre. Si l'on examine par exemple le degré de mathématisation du discours ${ }^{3}$, on constate non seulement que le discours de la physique est plus mathématisé que celui de la biologie qui, en revanche, a plus recours à des tableaux, diagrammes, etc., mais aussi que le sous-domaine peut à son tour faire varier ces paramètres : ainsi, le discours de la physique théorique apparaît plus mathématisé que celui de la physique " expérimentale », etc.

7 (b) La typologie discursive : on entendra par typologie discursive ce qui concerne l'appartenance d'une occurrence de discours, un texte ${ }^{4}$, à tel ou tel "genre " (Swales 1990), celui de l'article de recherche ou celui de l'abstract par exemple, pour citer les genres sans doute les plus connus et les plus étudiés, mais aussi celui de la demande de subvention, celui du rapport d'activité d'un laboratoire, celui du compte rendu de lecture (book review), celui de l'évaluation par un referee d'un article soumis pour publication, etc. La spécificité de chaque type se manifeste ici aussi dans un certain nombre de caractéristiques formelles : structuration d'ensemble et présentation matérielle, formules privilégiées, etc. On notera que ce facteur se situe sur un autre axe de structuration que le premier facteur (domanial) évoqué en (a) ci-dessus, mais que le premier axe de 
structuration reste pertinent. Ainsi, un abstract mathématique présentera-t-il à la fois des caractéristiques formelles du genre abstract et des caractéristiques formelles du domaine mathématique, dans une combinaison propre à le différencier d'autres abstracts et d'autres types de discours mathématique.

(c) La typologie textuelle : on entendra par typologie textuelle ce qui, à l'intérieur d'une occurrence de discours, un texte, concerne l'appartenance de telle ou telle partie de ce texte à tel ou tel type formel et/ou fonctionnel. On peut évoquer, pour prendre un exemple bien connu, les différentes parties du format «IMRAD » (Day 1989: 5-7 et passim ), ou songer aux différentes parties du discours mathématique : énoncés de théorèmes, de lemmes, démonstrations, etc. (Higham 1993 : 11-28 ; Petit 1993). La spécificité de chaque partie de texte se manifeste là encore dans un certain nombre de caractéristiques formelles. On précisera que cette typologie textuelle peut (doit) être élargie pour inclure le paratexte (titres, sous-titres, notes infrapaginales, etc.) et le péritexte (tableaux, diagrammes, etc., c'est-à-dire les éléments qui ne sont pas strictement textuels, mais entretiennent néanmoins un rapport essentiel avec le texte proprement dit). On notera là encore que ce nouvel axe de structuration de la diversité du discours scientifique vient se combiner avec les deux axes précédents.

(d) La situation de production : on entendra par situation de production ce qui concerne le ou les auteurs en tant que personne(s) (langue maternelle, statut professionnel, etc.) et en tant qu'instance, le processus d'écriture (à un ou à plusieurs, écriture en langue étrangère, aide rédactologique, etc.), le support de publication (nature et contraintes de la collection, de la revue, etc.), le destinataire, la date de publication, etc. On se trouve, avec ce dernier facteur de diversité, qui vient s'ajouter aux précédents, au niveau le plus spécifique, parce que le plus individuel.

Pour conclure cette section, nous postulons que la diversité du discours scientifique, dont le constat simple a été posé ci-dessus, peut légitimement être un objet d'étude de la stylistique, lorsqu'elle est envisagée dans l'ensemble de ses manifestations formelles combinées de façon unique dans chaque texte. Ce qui ne signifie pas qu'elle ne puisse pas être aussi un objet d'étude pour d'autres disciplines, qu'il s'agisse de sciences du langage (la diversité domaniale pourra par exemple être un élément déterminant d'une approche lexico-terminologique, la diversité discursive de l'analyse de genre, etc.) ou d'autres sciences humaines (la sociologie par exemple pourra s'intéresser à la pratique discursive des scientifiques). L'objet d'étude étant donc le style, tout ce qui précède tend à montrer qu'il n'y a pas, pour aborder la question du style dans l'étude du discours scientifique, un seul niveau, mais bien une pluralité de niveaux situés sur un parcours qui va du générique au spécifique, en termes de domaine, sous-domaine, spécialisation ; communauté, groupe, individu ; discours, texte, partie de texte ; mais aussi langage, langue, discours. On songe à une définition philosophique du style comme "modalité d'intégration de l'individuel dans un processus concret qui est travail» (Granger 1968:9). C'est la question de la langue qui va faire l'objet de la partie suivante.

\subsection{Le discours scientifique et l'anglais}

11 C'est un lieu commun que de rappeler le statut particulier de l'anglais comme langue de communication, notamment scientifique, internationale. Ce statut particulier a une conséquence matérielle dans le domaine français-anglais, que l'on peut vérifier facilement si l'on cherche à constituer un corpus scientifique contrastif (Petit 1993) : les 
traductions publiées du discours scientifique sont plus rares que celles d'autres types de discours, littéraire bien sûr, mais aussi journalistique, etc. Sans entrer dans le détail des constatations que l'on peut faire, il semble se dégager une tendance forte : les articles de recherche primaire et les ouvrages majeurs (traités d'enseignement supérieur et de recherche) d'auteurs francophones sont de plus en plus fréquemment publiés uniquement en anglais, soit qu'ils aient été rédigés directement en anglais avec une aide rédactologique professionnelle ou circonstancielle, soit qu'ils aient été traduits professionnellement à partir d'une version française de travail ${ }^{5}$. Dans tous les cas, il semble que cet état de fait doive conduire à s'interroger sur la méthodologie même d'une étude contrastive à vocation stylistique du discours scientifique ${ }^{6}$.

Il résulte en effet de ce qui précède qu'une partie du discours scientifique en anglais n'est pas écrite par des locuteurs natifs de l'anglais. Il n'est sans doute pas nécessaire de s'attarder ici sur ce qui se situe en amont de la publication, car cette question est bien connue des anglisticiens de spécialité. Rappelons simplement qu'elle fait l'objet de travaux de recherche spécifiques, qui en étudient divers aspects (Sionis, ici même), ainsi que de manuels, destinés à un public national particulier (Défourneaux 1980 pour les francophones), ou à un public international (Higham 1993: 51-66). Il faut en revanche, nous semble-t-il, s'attarder un peu sur l'aval, c'est-à-dire sur le statut qu'il convient d'accorder à ces textes une fois publiés ${ }^{7}$ pour l'étude du discours scientifique en anglais. On se trouve alors confronté à une série d'interrogations auxquelles on n'a peut-être pas encore accordé toute l'attention qu'elles méritent: Faut-il systématiquement exclure d'un corpus d'anglais scientifique tout texte de non-natif ${ }^{8}$ ? Que faire des textes signés de plus d'un auteur dont l'un au moins est anglophone ${ }^{9}$ ? Que faire des textes d'un auteur non anglophone, mais qui a vécu pendant un certain temps ou qui travaille régulièrement en pays anglophone ${ }^{10}$ ? etc. La liste est loin d'être exhaustive. Plutôt que de la poursuivre, on constatera qu'il résulte également de ce statut particulier de l'anglais qu'une partie (à préciser) du lectorat est, elle aussi, constituée de locuteurs non natifs de l'anglais. Cette double donnée ne peut être sans conséquence sur le discours scientifique. Le témoignage de nombre de scientifiques eux-mêmes va clairement dans ce sens, en ce qui concerne l'écriture en anglais langue étrangère, dont ils reconnaissent qu'elle peut modifier l'équilibre textuel de leurs productions et sans doute aussi les rapports entre la forme et le fond ${ }^{11}$. Il faudrait étudier aussi l'influence que peut avoir sur l'écriture des auteurs anglophones la nature (au regard du statut d'anglophone ou de non-anglophone) de leur lectorat. Certaines revues scientifiques et les guides d'écriture rappellent dans leurs recommandations ou instructions aux auteurs que leurs articles peuvent être lus par un public de non-anglophones, la seule conclusion semblant être que le style doit être d'autant plus clair :

The most fundamental tenet of technical writing is to keep your prose simple and direct. Much of written English is unnecessarily complicated. In writing up your research you are aiming at a relatively small audience, so it is important not to alienate part of it with long-winded or imprecise text. English may not be the first language of many of your readers - they, particularly, will appreciate plain writing. (Higham $1993: 2)^{12}$

On étudiera plus loin (2.2.) les incidences de cette idée de simplicité sur la conception du style scientifique.

Pour conclure sur ce point, nous pensons que le statut particulier de l'anglais, tel qu'il a été évoqué dans ce qui précède, constitue une donnée fondamentale, qui doit être prise en compte pour l'étude du discours scientifique en anglais, et notamment pour la 
définition d'une stylistique contrastive spécifique. Nous en venons maintenant à la question du style.

\section{Le style du discours scientifique}

\subsection{Le style peut-il être défini a priori?}

On commencera par une évidence : le concept de style apparait problématique, d'abord parce que le mot lui-même est polysémique. L'entrée style d'un dictionnaire général courant comme le Collins Dictionary of the English Language (1986) ne comporte pas moins de quatorze rubriques pour le seul substantif, dont les huit premières correspondent à des emplois non spécialisés ${ }^{13}$. Dans celles qui paraissent (plus ou moins directement) pertinentes pour la stylistique, on voit apparaître la notion de qualité avec un prolongement vers la notion d'élégance, l'opposition de la forme et du fond et la notion de caractéristiques envisagée en termes généraux ou particuliers. Mais le domaine de référence reste trop général, ou trop large (puisqu'il inclut la musique, la peinture, etc.), pour rendre ces définitions opératoires dans notre domaine. Il faut donc se tourner vers la littérature stylistique, et l'on constate immédiatement que le stylisticien se trouve confronté, dans son domaine spécialisé, à la même diversité que le lexicographe. L'une des premières tâches de tout ouvrage de stylistique consiste à tenter de donner à son tour une définition du concept de style, après avoir recensé et réévalué les définitions précédentes :

An analysis of some four or five score definitions of style culled from critical works, linguistic studies, dictionaries, and encyclopedias of different periods shows that such definitions can, allowing for much overlapping, be classified under a limited number of major headings. (Enkvist $1964: 10$ )

16 Cette classification même peut varier sensiblement d'un ouvrage à l'autre, mais il nous semble que la citation suivante peut résumer de façon assez représentative la diversité des définitions :

style as a shell surrounding a pre-existing core of thought or expression; as the choice between alternative expressions; as a set of individual characteristics; as deviations from a norm; as a set of collective characteristics; and as those relations among linguistic entities that are statable in terms of wider spans of text than the sentence. (Enkvist $1964: 12$ )

On voit qu'il est facile de critiquer l'hétérogénéité, le manque de précision ou le caractère aporétique ${ }^{14}$, de ces différentes approches ${ }^{15}$. Bref, des citations classiques ressassées ( «Le style est l'homme même») aux concepts contradictoires ou aux formulations (trop) techniques, par exemple

The style of a text is a function of the aggregate of the ratios between the frequencies of its phonological, grammatical and lexical items, and the frequencies of the corresponding items in a contextually related norm. (Enkvist $1964: 28)^{16}$,

les définitions du style construisent un ensemble complexe et souvent peu éclairant. On ne s'étonnera pas dans ces conditions que, pour certains stylisticiens, « la sagesse consiste donc à partir de la stylistique et non du style. On installe au départ une praxis, et on examine ce qu'on trouve à la fin » (Molinié 1986 : 9). Or, c'est bien l'installation même de cette praxis qui fait problème dans le cas du discours scientifique et il faut donc, sans inverser la démarche, en passer d'abord par quelques constatations d'expérience. 


\subsection{L'expérience du style dans le discours scientifique}

19 Nous nous intéressons ici à l'expérience globale de l'anglisticien de spécialité qui étudie le discours scientifique en anglais, et le pratique à des titres divers (comme objet d'enseignement, de recherche, d'activité rédactologique ${ }^{17}$, etc.). Le point de vue n'est donc pas strictement, à ce stade, celui d'une approche stylistique raisonnée, mais on accompagnera les constatations elles-mêmes de quelques réflexions permettant d'avancer dans cette direction.

20 (a) Il existe un ensemble de caractéristiques génériques dont la perception intuitive permet de reconnaître immédiatement une occurrence discursive (un texte) comme relevant du discours scientifique et du style scientifique. On sera conduit à faire ici deux remarques.

La première remarque est pour souligner le caractère indissociable de ce que l'on pourrait appeler, par commodité, la forme et le fond, et donc le caractère global de cette perception. Ainsi, il ne suffit pas qu'un texte traite d'un sujet d'intérêt scientifique, et donc comporte un lexique spécialisé, pour qu'il soit perçu comme relevant du discours et du style scientifiques : il peut en effet relever du discours et du style journalistiques ${ }^{18}$, voire littéraires ${ }^{19}$. Il ne suffit pas non plus qu'il mette en oeuvre certaines caractéristiques formelles, telles que symboles scientifiques, diagrammes, schémas, etc.: on connaît l'usage qu'en fait par exemple le discours de la publicité. On conçoit donc que le style scientifique ne saurait être défini par une liste de caractéristiques (terminologie spécialisée, utilisation de notations symboliques, etc.) dont la présence ou l'absence serait un critère suffisant de détermination.

La deuxième remarque concerne le discours des sciences et non plus le discours scientifique. Le constat, dressé plus haut, de la diversité du discours scientifique devrait conduire à parler au pluriel des styles scientifiques. Or, la pratique ne paraît pas confirmer ce passage au pluriel : on rencontre en effet plus de références à "la langue » de la chimie, de la biologie, de l'océanographie, etc. ${ }^{20}$, qu'au « style » de la chimie, etc ${ }^{21}$. La spécificité domaniale est, semble-t-il, perçue plus comme une question d'ordre lexicoterminologique que stylistique, la spécificité du lexique d'une spécialité ne suffisant pas à fonder un style à part entière. On peut peut-être voir, en empruntant une comparaison au domaine de la sociolinguistique et des études de la variation, dans le style (du discours) de la chimie, de la biologie, etc., les variétés d'un même style scientifique (sur le modèle des rapports entre dialectes et langue $)^{22}$.

(b) Un texte relevant du discours scientifique peut présenter, outre les caractéristiques génériques, et les autres caractéristiques qui marquent son appartenance à tel ou tel domaine ou genre, évoquées en (a) ci-dessus, un ensemble de caractéristiques plus spécifiques qui ressortissent au niveau individuel.

Il faut, avant même de donner quelques exemples, se demander si cette dimension est pertinente pour une étude du discours scientifique, dont les objectifs, à la différence de ceux d'une étude littéraire, ne semblent pas inclure de façon nécessaire la caractérisation de l'écriture de tel ou tel auteur. La question se pose d'autant plus que nombre d'ouvrages ou articles scientifiques sont écrits en collaboration, qu'ils font l'objet d'un processus de mise au point définitive qui comporte plusieurs phases de relecture et modification, etc. Surtout, cette dimension individuelle parait en contradiction flagrante avec l'idée bien connue selon laquelle le style scientifique est, et doit être impersonnel, neutre, 
transparent, etc. En voici une formulation qui, reprenant une idée assez répandue (pas seulement dans l'esprit des scientifiques), tend à définir le style scientifique comme non littéraire :

A scientific paper is not literature. The preparer of a scientific paper is not really an author in the literary sense.

Some of my old-fashioned colleagues think that scientific papers should be literature, that the style and flair of an author should be clearly evident, and that variations in style encourage the interest of the reader. I disagree. (Day $1989: 12$ )

On reviendra sur ce point en (c) ci-dessous.

Pour prendre maintenant un exemple précis, l'emploi de la conjonction For ${ }^{23}$ («car») paraît peu fréquent dans le discours mathématique en anglais, mais certains auteurs l'utilisent régulièrement, à la fois dans les parties de commentaire :

Returning now to the special case of homotopy of paths, it turns out that the concept defined above is not of great interest unless some further restriction is imposed. For it is not hard to show that any two paths on an arcwise connected space are homotopic. (Wallace $1957: 66)^{24}$

et dans les parties de démonstration :

These two necessary conditions for the equivalence of the property $C$ to continuity are also sufficient. For suppose that they are both satisfied, and let $f$ be a real valued function continuous at $p$. Then... (Wallace $1957: 11)^{25}$

Cette caractéristique est d'autant mieux identifiable qu'elle est récurrente chez l'auteur visé et rare chez les autres auteurs. Encore faut-il, pour dépasser le stade de la simple impression, se donner les moyens de mettre les auteurs, et leurs textes, en perspective, à la fois diachronique et synchronique. Sur la question de l'emploi de For dans le discours mathématique, cette mise en perspective diachronique semble indiquer que, s'il a à peu près disparu dans les textes récents et actuels, il était encore assez répandu dans les textes de la première moitié du siècle, mais que, à la période qui correspond aux exemples cités, son emploi régulier était déjà « marqué $»^{26}$.

Plus fondamentalement, il faut noter qu'une caractéristique locale quelconque, même si son statut de caractéristique est avéré, n'a pas en soi une dimension stylistique. Il paraît clair en effet que, pour la définition du style individuel comme pour les autres niveaux auxquels on peut appréhender le style, c'est un ensemble de caractéristiques qui doit être considéré et c'est cet ensemble qui peut permettre d'établir un profil stylistique (Crystal 1991 ; Petit 1995).

(c) Un texte relevant du discours scientifique peut, comme n'importe quel autre texte, faire l'objet d'un jugement qualitatif dans les termes habituels : bien ou mal écrit, style clair ou confus, élégant ou maladroit, etc.

Cette approche de la qualité du style concerne là encore une perception intuitive globale. Les recommandations des professionnels du discours scientifique insistent toutes sur les idées de simplicité, de clarté, voire de transparence. On citera ici un extrait de l'une des contributions à l'ouvrage intitulé How to write mathematics commandé par l'American Mathematical Society à un petit groupe d'éminents mathématiciens :

I should like to argue that it is important that mathematical books (and papers, and letters, and lectures) be written in good English style, where good means " correct » according to currently and commonly accepted public standards. (French, Japanese, or Russian authors please substitute "French", "Japanese", or "Russian» for «English ».) I do not mean that the style is to be pedantic, or heavy-handed, or formal, or bureaucratic, or flowery, or academic jargon. I do mean that it should be completely unobtrusive, like good background music for a movie, so that the reader may 
proceed with no conscious or unconscious blocks caused by the instrument of communication and not its content. (Halmos $1973: 33)^{27}$

L'objet visé par ces recommandations est ici le style mathématique, mais la généralisation au style scientifique paraît légitime. La même idée revient en effet dans nombre de guides d'écriture scientifique, qui dénoncent les sources possibles d'obstruction dans la transmission du message :

Scientific writing is the transmission of a clear signal to a recipient. The words of the signal should be as clear and simple and well-ordered as possible. In scientific writing, there is no room for and no need for ornamentation. The flowery literary embellishments, the metaphors, the similes, the idiomatic expressions, are very likely to cause confusion and should seldom be used in writing research papers. [...] Literary tricks, metaphors and the like, divert attention from the substance to the style. They should be used rarely, if at all, in scientific writing. (Day $1991: 2-3)^{28}$

La principale qualité du style scientifique, aux yeux de ses praticiens, est donc d'être en quelque sorte un degré zéro du style. Mais au-delà de ces considérations générales, comment évalue-t-on l'adéquation du style d'un texte à cet idéal du style scientifique ?

La question se pose non seulement en termes théoriques mais aussi en termes pratiques car, si le jugement qualitatif reste souvent implicite dans le cas du lecteur ordinaire, il peut néanmoins devoir être formulé explicitement dans le cas du lecteur spécialisé (membre du comité de lecture, referee, relecteur rédactologue, etc.). La difficulté apparaît alors grande de détailler les composantes du phénomène : ainsi, le lecteur spécialisé qui aura formulé tel jugement d'ensemble, par exemple "style parfois confus », pourra-t-il signaler quelques exemples locaux de cette confusion, du type de ceux qui sont répertoriés dans les guides d'écriture :

It can be ambiguous: in the sentence "Condition 3 is not satisfied for the steepest descent method, which is why we do not consider it further", we cannot tell whether it is the condition or the method that is not being pursued. (Higham 1993: 34), précis. Les guides d'écriture là encore fournissent de nombreux exemples de ces conseils susceptibles d'améliorer le style :
4.21. Omit These Words?
Here are some words and phrases whose omission often improves a sentence:
actually, very, really, currently, in fact, thing, without doubt. (Higham $1993: 41$, Ch
$4:$ « English Usage ») $)^{29}$
4.26. Sentence Opening
Try not to begin a sentence with there is or there are. These forms of the verb be make a weak start to a sentence [...]. Sometimes these phrases can simply be deleted, as in the sentence "There are several methods that are applicable" ("Several methods are applicable"). Also worth avoiding, if possible, are "It is" openers, such as "It is clear that" and "It is interesting to note that". If you can find alternative wordings, your writing will be more fresh and lively. (Higham 1993: 44, Ch. $4:$ « English Usage »)

Ils fournissent aussi un ensemble de considérations très (trop) générales, dont la remarque suivante est représentative :

Good writing reflects clear thinking. It is very hard for a woolly thinker to produce clear prose. But clear thinking is only a necessary, not a sufficient, condition for good writing. Clear thinking leads to good organization, a vital ingredient of technical writing. A well-organized piece is much easier to write than a badly organized one. If you find a particular piece difficult to write it may be because you 
have not found the right structure to express your ideas. (Higham $1993: 2$, Ch. 1 :

«General Principles »)

\section{Darbelnet (1972). L'ambition stylistique en est explicite et leur cadre théorique de départ} reprend la distinction de Bally entre deux types de stylistique :

L'une cherche à dégager les moyens d'expression d'une langue donnée en opposant les éléments affectifs aux éléments intellectuels. C'est la stylistique interne. L'autre s'attache à reconnaître les démarches des deux langues en les opposant l'une à l'autre. Nous l'appellerons la stylistique comparative externe, ou stylistique comparée. [...]

Reprenant maintenant notre distinction entre servitude et option, nous dirons que, si les options dominent dans la stylistique interne, qui étudie surtout les faits d'expression, la stylistique externe traite à la fois de servitude et d'option. (32-33)

Si le programme est séduisant, la réalité de cet ouvrage classique et fondateur paraît en décalage par rapport à ses ambitions. On constate en effet que, dans le détail des analyses et conclusions, l'accent est mis sur une forme de stylistique que l'on pourrait dire, le mot " tendance » revenant fréquemment, tendancielle. Elle conduit, nous semble-t-il, à perdre de vue ce que les auteurs appellent «les options», au profit de tendances privilégiées, « tendance de l'anglais à se calquer sur le réel » $(150)^{32}$, « tendance du français d'annoncer plus tôt que l'anglais la démarche de sa pensée » $(225)^{33}$, « prédominance du substantif en français » alors que l'anglais préfère « la tournure verbale » (102-103), etc. Ces tendances, précisent Vinay et Darbelnet, ne sont pas fortuites mais correspondent au "génie » de chaque langue (258). Il est assurément facile de critiquer cette stylistique tendancielle sans prendre en compte les objectifs propres de l'ouvrage, que le sous-titre, rappelons-le, présente comme une Méthode de traduction, mais, même avec cet objectif défini, on voit 
bien le côté insatisfaisant d'une généralisation comme celle qui est exprimée dans la citation ci-dessous :

Le français, tout au moins dans la langue littéraire, philosophique, scientifique et juridique, affectionne les articulations, et se passe difficilement des précisions qu'elles peuvent apporter dans le déroulement de la pensée. L'anglais au contraire, même dans ses formes classiques, fait beaucoup moins appel aux articulations explicites, donc laisse au lecteur le soin de suppléer lui-même les articulations qui s'imposent et joue plutôt avec la juxtaposition des phrases et segments de l'énoncé. $(222)^{34}$

Pour le discours scientifique en tout cas, pourtant explicitement visé par cette remarque, une telle généralisation paraît pour le moins discutable et il suffit d'ouvrir un texte scientifique pour s'en convaincre. Examinons néanmoins un exemple :

The theory of the last section will now be illustrated by application to a number of simple cases. It will appear that the use of the exact sequence (28) along with a certain amount of ingenuity suffices to calculate the homology groups of some of the most elementary spaces. It will also become clear, however, that some more systematic method is necessary to deal with spaces which present even the mildest complications. (Wallace 1957 : 153)

Nous allons maintenant illustrer la théorie du paragraphe précédent en l'appliquant à un certain nombre de cas simples. Il apparaîtra que l'usage de la suite exacte (28), joint à une certaine dose d'ingéniosité, suffit pour calculer les groupes d'homologie de quelques-uns des espaces les plus élémentaires. Il apparaîtra pourtant clairement que des méthodes plus systématiques sont nécessaires pour s'occuper d'espaces qui présentent des complications même très "gentilles". (Wallace 1957, trad. Verley $1973: 173$ )

Rien ne paraît confirmer ici ces tendances respectives qui feraient du français une langue à "mode de développement raisonné », alors que l'anglais aurait un «mode de développement intuitif ou sensoriel » (Vinay et Darbelnet 1972 : 220-222). On remarquera que la traduction ci-dessus semble même plutôt affaiblir certaines articulations, par exemple dans la traduction de It will also become clear, however, où l'on note la disparition de also, et l'on constatera aussi que cet exemple contredit la tendance nominale du français qui se distinguerait ainsi de l'anglais plus verbal : by application traduit par « en l'appliquant». On peut penser que, dans ce cas précis, c'est la qualité de la traduction qui est en cause..$^{35}$ Mais au-delà même de cette question méthodologique cruciale pour toute approche contrastive, sur laquelle on sera conduit à revenir, il nous semble que cette stylistique tendancielle, parce que précisément elle a l'ambition de fonder une stylistique sur les seules tendances dues au " génie » de chaque langue, se trouve dans l'incapacité de rendre compte de la spécificité proprement stylistique des usages du langage à travers les deux langues comparées et dans les textes. Il serait fastidieux de multiplier les exemples, mais on montrerait facilement que nombre des tendances recensées par la stylistique comparée peuvent être également prises en défaut.

L'analyse contrastive, illustrée notamment par les travaux de Guillemin-Flescher, a un tout autre objectif que la stylistique comparée. Il ne s'agit pas de stylistique, ni d'une méthode de traduction, mais de linguistique contrastive, c'est-à-dire de linguistique dont le but est de « dégager les opérations qui sous-tendent l'activité langagière et la façon dont elles se réalisent en français et en anglais » (Guillemin-Flescher 1981 : vii). L'analyse se fait sur un corpus bilingue (texte et traduction), dans un cadre théorique particulier qui est celui de la théorie des opérations énonciatives de Culioli. Nous connaissons bien, pour avoir pratiqué nous-mêmes cette approche dans notre travail de thèse (Petit 1993), sa pertinence analytique pour le discours scientifique. Nous en sommes cependant venu à 
ressentir, à la fois comme linguiste contrastiviste et comme anglisticien de spécialité, le besoin de prendre en compte ce qui n'était pas des récurrences systématisables, fondement de l'analyse contrastive, et de nous donner les moyens d'en rendre compte (Petit 1995 : 77-78). Pour illustrer ce point, nous reprendrons l'exemple de l'emploi de For $\left(\right.$ «car $\left.{ }^{36}\right)$ dans le discours mathématique en anglais. Une analyse proprement contrastive, à travers une étude minutieuse des différentes traductions auxquelles ce marqueur peut donner lieu en français ("car», "en effet", "ainsi», zéro, autres traductions $)^{37}$, serait notamment conduite à analyser la relation discursive en anglais et en français (relation interphrastique ou interpropositionnelle, repérage, thématisation, etc.). Cette étude, indispensable, laisse un point essentiel dans l'ombre : le choix de For dans le discours anglais de départ, s'il est envisagé en tant qu'opération linguistique, et peut ainsi être contrasté avec d'autres structurations, correspondant à des opérations linguistiques différentes, ne nous paraît pas l'être en tant que choix individuel effectif (attesté) entre les divers possibles. C'est cette dimension, qui fait intervenir d'autres considérations, comme celles que nous avons présentées dans les deux premières parties de notre réflexion, que nous appelons stylistique. Il en va de même du choix, pour chaque traduction, entre les divers possibles. Pour conclure sur ce point, nous pensons que la prise en compte de cette dimension stylistique contrastive doit se fonder sur une approche variationnelle, suivant l'expression qui donne son sous-titre à l'ouvrage de Hewson \& Martin (1991), auquel nous renvoyons pour un exposé détaillé.

\subsection{Stylistique, linguistique et discours scientifique}

Avant même que de s'interroger sur les rapports de la stylistique et de la linguistique, il ne nous paraît pas inutile de rappeler, même si c'est une évidence, que le discours scientifique peut être un objet d'étude pour d'autres disciplines que celles des sciences du langage: nous avons mentionné plus haut la sociologie, mais on pourrait mentionner aussi la philosophie, l'histoire des sciences, etc.

Pour rester dans le domaine des sciences du langage, au sens large, on voit bien que le discours scientifique peut constituer un objet d'étude pour l'analyse de discours, la grammaire de texte, l'analyse de genre, la traductologie, la pragmatique, la lexicologie et la terminologie, etc. Si aucune de ces approches n'est proprement stylistique, il nous paraît manifeste que toutes peuvent apporter des résultats pertinents pour la stylistique, qu'il s'agisse, pour reprendre la distinction essentielle rappelée dans ce colloque par Gilles Mathis, d'analyse stylistique ou d'étude de style. La stylistique apparaît alors comme une discipline dont l'objet est précisément de montrer comment l'ensemble des données linguistiques mises à jour par les différentes disciplines linguistiques forme une combinaison unique, à quelque niveau qu'on l'étudie, celui du discours, du texte ou de l'auteur. C'est d'ailleurs cette vocation de synthèse qui, à nos yeux, en fait une véritable discipline linguistique. Il nous semble, par ailleurs, aller de soi que ce caractère unique de chaque combinaison rend toute stylistique nécessairement contrastive, ou différentielle. On peut sans doute en tirer des conclusions quant à la problématique fameuse de la norme et de l'écart, mais il s'agirait là de théorie stylistique, qui est donc l'affaire des théoriciens, et notre approche ne se veut que de pratique raisonnée.

Il resterait à montrer cette pratique à l'oeuvre, ce que le format de cette étude ne permet que d'esquisser ici. Soit les deux exemples suivants : 
[1] 2. Definition and examples of $n$-manifolds

Assume $n$ is a positive integer. An $n$-dimensional manifold is a Hausdorff space (i.e., a space that satisfies the T2 separation axiom) such that each point has an open

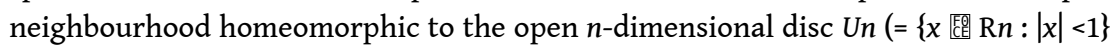
). Usually we shall say " $n$-manifold" for short. (Massey $1967: 2$ )

[2] Definition 3.4.1. A Hausdorff space is called an $n$-manifold if each point of $M$ has a neighbourhood homeomorphic to an open set in $\mathbf{R} n$.

Notice that any space homeomorphic to an $n$-manifold is itself an $n$-manifold, as also is any open subset of an $n$-manifold. (Maunder $1980: 88$ ) mathématique en matière de style des définitions, ni à une spécificité stylistique de la façon de l'un ou de l'autre auteur d'écrire les définitions, etc. Si l'on poursuit l'investigation dans la direction suggérée par la simple observation (l'espace de Hausdorff n'est pas «traité » de la même manière dans les deux définitions) renforcée par la linguistique (en termes de thématisation), on peut vérifier que, en [2], non seulement l'espace de Hausdorff a effectivement été introduit et défini précédemment, mais aussi que sa thématisation " prépare » un argument de la suite (une page plus loin) :
... The last example shows the reason for insisting on the Hausdorff space in Definition

3.4.1.: we wish to exclude such freak spaces. (Maunder : 89) ${ }^{39}$

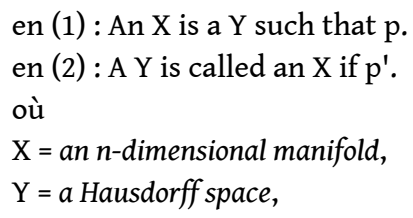

$p$ et $p^{\prime}$ ayant, pour autant que nous puissions en juger, le même contenu propositionnel, que nous ne nous hasarderons pas à reformuler.

On ne peut évidemment en tirer une conclusion quant à une tendance de l'anglais nous semble que la mise en regard de ces deux seuls exemples suggère un point d'entrée précis dans le style mathématique (analyse stylistique de la thématisation dans les définitions mathématiques) qui est susceptible de conduire à une investigation stylistique d'envergure incluant aussi la dimension individuelle ${ }^{40}$, et se prêtant bien sûr également à une comparaison entre les deux langues. On conçoit que d'autres exemples permettraient de suggérer d'autres points d'entrée (par exemple analyse stylistique des modulateurs d'assertion [hedges] dans le discours scientifique) également susceptibles de conduire à une mise en oeuvre de cette stylistique contrastive. 


\section{Conclusion} niveau et à explorer les perspectives ouvertes par Gilles-Gaston Granger ${ }^{41}$ qui envisag dans son Essai d'une philosophie du style, « une stylistique de la science, présentée comme un premier essai d'une stylistique générale » $(16)^{42}$. L'ambition de notre étude est, faut-il le dire, infiniment plus restreinte. Notre réflexion, qui n'est pas celle d'un théoricien de la stylistique, se veut simplement réflexion raisonnée sur les orientations d'une pratique spécifique appliquée au domaine de l'anglistique et à l'étude des discours spécifiques. Nous espérons néanmoins que, au-delà de la mise au point méthodologique de cette pratique appliquée, elle pourra contribuer à la théorie de l'anglais de spécialité.

\section{Références du corpus}

\section{BIBLIOGRAPHIE}

Crystal, D. 1991. «Stylistic Profiling ». In Aijmer \& Altenberg (dir.), English Corpus Linguistics.

Londres : Longman, 220-238.

Crystal, D. \& D. Davy. 1969. Investigating English Style. Londres : Longman.

Day, R. A. 1989. How to Write \& Publish a Scientific Paper. Cambridge : Cambridge University Press.

Défourneaux, M. 1980. Do you speak science? Paris : Gauthier-Villars.

Dubois, J. et al. 1994. Dictionnaire de linguistique et des sciences du langage. Paris : Larousse.

Enkvist, N. E., J. Spencer \& Gregory. 1964. Linguistics and Style. Londres : OxfordUniversity Press.

Granger, G.-G. 1968. Essai d'une philosophie du style. Paris : Armand Colin.

Guillemin-Flescher, J. 1981. Syntaxe comparée du français et de l'anglais. Gap : Ophrys.

Halmos, P. R. In Steenrod et al. 1973, 19-48. ${ }^{43}$

Hewson, L. \& J. Martin. 1991. Redefining translation - The Variational Approach. Londres : Routledge.

Higham, N. J. 1993. Handbook of Writing for the Mathematical Sciences. Philadelphie : Siam (Society for Industrial and Applied Mathematics).

Molinié, G. 1986. Eléments de stylistique française. Paris : Presses Universitaires de France.

Petit, M. 1993. « Analyse contrastive de la relation discursive et inter-énonciative dans un corpus d'ouvrages mathématiques en français et en anglais ». Thèse de doctorat, Université Paris 7.

Petit, Michel. 1995. « De la pertinence du 'stylistic profiling'pour l'étude du discours de spécialité ». Bulletin de la Société de Stylistique anglaise 16, 67-80.

Steenrod, N.E. et al. 1973. How to Write Mathematics. American Mathematical Society.

Swales, J. M. 1990. Genre Analysis. English in academic and research settings, Cambridge : Cambridge University Press. 
Vinay, J.-P. et J. Darbelnet. 1972. Stylistique comparée du français et de l'anglais. Paris : Didier.

\section{Bibliographie complémentaire}

Anscombre, J-C. (dir.). 1995. Théorie des topoï. Paris : Ed. Kimé.

Beacco, J-C. 1994. « Données multilingues et description des textes : enjeux théoriques ». In

Moirand, S. et al. (dir.), Parcours linguistiques de discours spécialisés. Berne : Peter Lang, 263-270.

Groupe $\mu 1970$ [1982]. Rhétorique générale. Paris : Seuil.

Kaplan, R.B. 1983. «Contrastive rhetorics : Some implications for the writing process ». In Freedman et al. (dir.), Learning to Write: First Language and Second Language. Londres : Longman.

Lerat, P. 1995. Les langues spécialisées. Paris : Presses Universitaires de France.

Molinié, G. 1991. La stylistique, 2e éd. corr. « Que sais-je ?». Paris : Presses Universitaires de France.

Reboul, O. 1993. La rhétorique, 4e éd. corr. « Que sais-je ? », « Que sais-je ? ». Paris : Presses Universitaires de France.

Suhamy, H. 1993. Les figures de style. 6e éd, « Que sais-je ?». Paris : Presses Universitaires de France.

Suhamy, H. 1994. Stylistique anglaise. Paris : Presses Universitaires de France.

Ullmann, S. 1966. Language and Style. Oxford : Basil Blackwell.

Weissberg, R. \& S. Buker. 1990. Writing Up Research. Englewood Cliffs, NJ : Prentice Hall.

Massey, W. S. 1967. Algebraic Topology: An Introduction. New York : Springer Verlag.

Maunder, C.R.F. 1980. Algebraic Topology. Cambridge : Cambridge University Press.

Wallace, A. H. 1957. An Introduction to Algebraic Topology. Oxford : Pergamon.

Wallace, A. H. 1973. Introduction à la topologie algébrique. Trad. par J.-L. Verley Paris : GauthierVillars.

\section{NOTES}

1. On en trouvera des échos dans l'article «Stylistique » de Dubois et al. (1994).

2. Ce qui vaut ainsi pour le discours scientifique vaut, naturellement, pour tout autre discours spécifique, le discours littéraire lui-même pouvant/devant en ce sens être perçu comme un discours spécifique parmi d'autres.

3. Que l'on peut définir grossièrement par l'utilisation de notations symboliques et de raisonnements formels sur ces notations.

4. La notion de texte mériterait d'être précisée. On se contentera ici d'une approche intuitive : un texte est ce qui forme un tout et est perçu comme tel. En ce sens, l'abstract, évoqué plus loin, peut être considéré comme un texte, puisqu'il peut exister indépendamment de l'article qu'il résume (dans certaines revues par exemple les abstracts sont regroupés dans une section séparée).

5. Il n'existe pas, à notre connaissance, d'étude complète sur ce point.

6. On utilise ici à dessein le terme "étude contrastive » pour marquer sa différence avec les objectifs et les méthodes de l'analyse contrastive, que l'on évoquera en troisième partie. 
7. On suppose que, à ce stade, les fautes d'anglais (de morpho-syntaxe essentiellement) et les maladresses flagrantes (celles qui entravent la compréhension) ont été corrigées. Il s'agit donc d'un anglais « correct », et toute la question tourne autour de son authenticité.

8. Question subsidiaire : Ne faut-il pas faire une distinction entre l'anglais langue étrangère et l'anglais langue seconde?

9. Question subsidiaire: Comment déterminer avec précision le rôle exact de chacun des signataires, et donc celui de l'anglophone?

10. Question subsidiaire : Comment traiter le cas des auteurs qui, par exemple, sans avoir vécu aux États unis, ou sans y enseigner tous les ans un mois ou deux, sont néanmoins en contact régulier, dans leur laboratoire de recherche et dans les manifestations scientifiques, avec des anglophones?

11. Les participants au XVIe Colloque du GERAS (Évry, 1995) se rappelleront la table ronde plénière avec des scientifiques et les propos de la collègue mathématicienne invitée reconnaissant que, en anglais, elle écrivait les mathématiques de façon différente, avec moins d'explications, justifications ou commentaires rédigés (à cause de la difficulté de la langue) et plus de «formules».

12. C'est nous qui soulignons.

13. D'autres concernent par exemple la botanique ou la zoologie.

14. On connaît par exemple les débats autour de la notion de norme et de déviation.

15. Pour une critique détaillée, voir Enkvist (1964: 10-27).

16. Il n'était que justice de citer la propre définition d'Enkvist après avoir eu recours à lui pour ce qui précède, mais faut-il avouer que cette définition nous laisse perplexe?

17. On exclura à nouveau à cet égard de l'expérience rédactologique de l'anglisticien ce qui concerne la correction morpho-syntaxique.

18. La question de la vulgarisation scientifique est bien connue des enseignants-chercheurs en anglistique de spécialité.

19. On songera par exemple aux chapitres encyclopédiques de Moby Dick sur les cétacés.

20. Ou encore, dans un contexte d'anglais langue étrangère, à « l'anglais » de la chimie, etc.

21. Avec une exception sans doute pour le "style mathématique», peut-être parce que l'outil mathématique et certains de ses modes discursifs sont utilisés par d'autres sciences et que l'adjectif «mathématique " se prête bien à la collocation (à la différence par exemple de " chimique »).

22. On notera, pour appuyer indirectement cette comparaison, que le domaine de spécialité est parfois appelé dans la littérature "province». On peut penser en outre que, si l'on s'attachait à pousser la comparaison, on pourrait certainement utiliser avec profit des concepts sociolinguistiques comme ceux d'isoglosse et de faisceau d'isoglosses, etc.

23. Avec une majuscule car, dans cet emploi, toujours en tête de phrase. On verra plus loin (3.1.) la différence avec le français.

24. C'est nous qui soulignons.

25. C'est nous qui soulignons.

26. Il faudrait bien évidemment une étude de plus grande envergure sur corpus pour dégager des résultats plus précis sur ce point.

27. C'est nous qui soulignons.

28. C'est nous qui soulignons.

29. La rubrique est reproduite in extenso. On comprend facilement que les adverbes ou syntagmes prépositionnels puissent être simplement supprimés, mais qu'en est-il de l'omission de thing?

30. Signalons par exemple, sur un sujet bien connu en anglistique de spécialité, la rubrique suivante : « 4.4. Active versus Passive - Prefer the active to the passive voice (prefer " $X$ did $Y$ " to "Y was done by X"). The active voice adds life and movement to writing, whereas too much of the passive voice weakens the communication between writer and reader. » (Higham 1993 : 31) 
31. On trouvera, pour les mathématiques, des listes de «Winners of Prizes for Expository Writing » dans Higham (1993 : 193-209). Il serait intéressant d'étudier ces textes à la lumière des préceptes des guides d'écriture mathématique.

32. À propos de «l'aspect successif » (ex. : mile upon mile, wave after wave,...).

33. À propos des charnières.

34. C'est nous qui soulignons.

35. Et la traduction de la dernière phrase de l'exemple ci-dessus (even the mildest complications) n'aura à cet égard pas manqué d'attirer l'attention.

36. Sans majuscule, car en tête de proposition et non pas de phrase comme For.

37. Toutes traductions recensées dans Wallace (1957, trad. Verley 1973), qui pourrait constituer un élément de corpus. Il faudrait bien sûr préciser les autres modifications de l'énoncé dans chaque cas.

38. "This textbook is designed to introduce advanced undergraduate or beginning graduate students to algebraic topology " (Massey : ix). "Most of the book is based on lectures to thirdyear undergraduate and first-year postgraduate students » (Maunder : v).

39. C'est nous qui soulignons.

40. On peut par exemple identifier et expliquer des cas de thématisation mal maîtrisée et fonder ainsi un jugement qualitatif sur le style.

41. Dont nous rappelons la définition citée plus haut du style comme « modalité d'intégration de l'individuel dans un processus concret qui est travail » (1968: 9).

42. La première partie est intitulée « Le style dans la construction de l'objet mathématique ».

43. Les contributions individuelles à cet ouvrage n'ont pas de titre individuel.

\section{RÉSUMÉS}

On postule que la stylistique a un rôle important à jouer dans l'étude du discours scientifique. Après avoir examiné les facteurs de diversité du discours scientifique et le statut de l'anglais scientifique, on s'intéresse à la question du style scientifique, notamment en termes d'expérience commune. On est enfin conduit, en étudiant les approches contrastives, à envisager une stylistique véritablement contrastive propre à rendre compte des spécificités du discours scientifique.

Our initial postulate is that stylistics can play a major part in the study of scientific discourse. We first examine the various factors which contribute to the diversity of scientific discourse, together with the special role of English. We then look into the question of scientific style in the light of experience. Finally we review other contrastive approaches and envisage a really contrastive form of stylistics capable of accounting for the specificities of scientific discourse.

\section{INDEX}

Mots-clés : discours scientifique, linguistique contrastive, mathématiques, style, stylistique Keywords : contrastive linguistics, mathematics, scientific discourse, style, stylistics 
AUTEUR

MICHEL PETIT 